\title{
Automatic Feedback Control of Woolen Card
}

\author{
By Fumio Murakami, Susumu Kase and Toshio Shoman, Members, TMS,
}

Toyo Spinning Co., Ltd., Osaka.

\begin{abstract}
A series of field tests was made on feedback control equipment mounted on a woolen card to obtain a mathematical model describing its dynamic characterisrics. Input signals to the control system, including the random noise generated by the photoelectrical measurement of web thickness, were then analyzed with the aid of statistical method to relate the above mentioned mathematical model to the actually measured values of roving weight variation.

It was found that the statistical methods of automatic control were highly useful for an accurate analysis of yarn-evening devices.
\end{abstract}

\section{Introduction}

The authors' primary objective in making the tests under review was to develop a reasonably accurate and reasonably quick method to evaluate the performance of yarn weight-evening devices on mill site.

The automatic control equipment tested was a commercially available unit known by the trade name W.I.R.A./B.E.P. Autocount Wool Carding Control Equipment and developed by the Lancashire Dynamo Electronic Products Ltd., England. The control equipment had been in commercial operation at the Thushima mill of Toyo Spinning Co., Ltd., for about three years before the experiments under review were made.

As shown in Fig. 1, the control equipment has a photoelectric measuring element which measures the thickness of the web leaving the doffer by sensing the intensity of a light flux that passes through the web twice. The intensity of the light source is kept constant by an electronic servo contained in the measuring element.

Note here that only $9 \%$ of the total width of the web (or the equivalent of 12 yarn ends out of a total of 144) is covered by the measuring element.

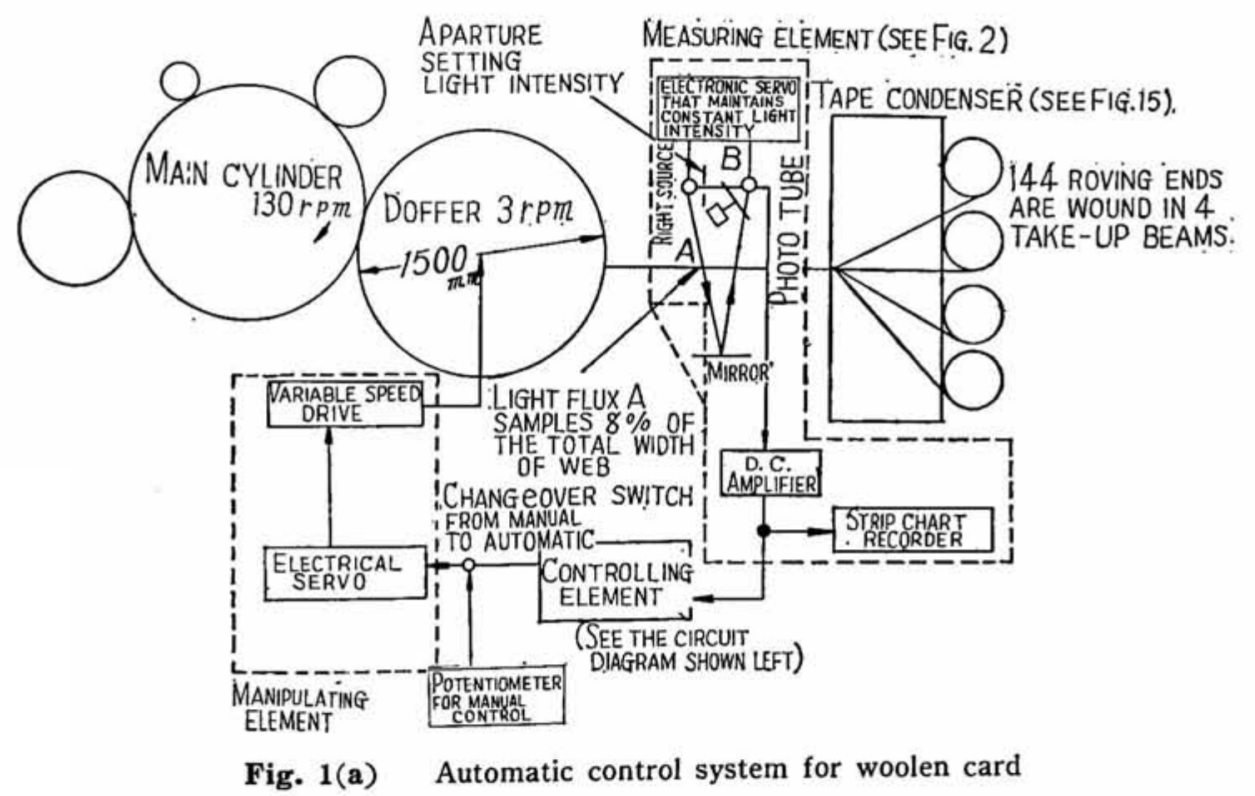


High gain ampfier circuit in the controlling element

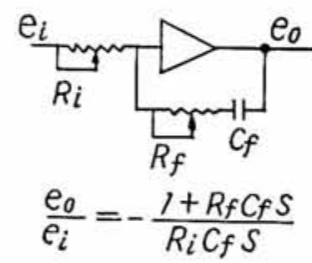

Transfer function of controlling element

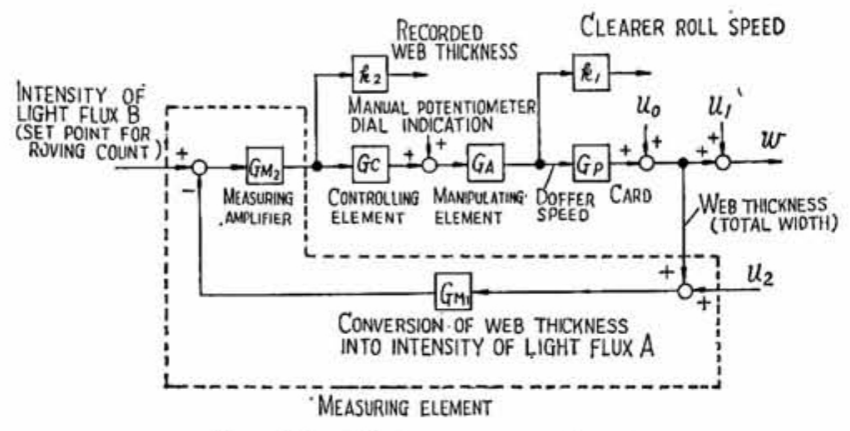

(See Fig. 16 for $u_{0}, u_{1}$ and $u_{2}$ )

Fig. 1(b) Woolen card control system expressed in block diagram

In other words, the control system is made to take a $9 \%$ sample out of the controlled variable. The significance of this sampling will be discussed later in relation to the statistical analysis of random noise in the control system.

Measured web thickness in the form of D.C. voltage then enters a strip-chart recorder and the controlling element.

The controlling element shown on the left-hand side of Fig. 1(a) is essentially a proportional plus reset controller which drives an electrical servo con- sisting of a two-phase motor and gas-filled tubes

Driven by the servo, in turn, is a variable speed drive which changes the doffer speed in proportion to the output voltage of the controlling element. To reduce roving weight variation, the control equipment naturally increases the doffer speed when the measured web thickness exceeds the desired value and vice versa.

The most technically sophisticated component in the woolen card control system is the photoelectric measuring element. The ingenuity of its design lies in the fact that one photo tube and one light source serve both to measure web thickness and to maintain a constant intensity of light source. Such circuit keeps the measuring element stable and free of zero drifts over an indefinite length of time.

As shown in Fig. 1(a), light emitted by the light source reaches the photo tube by way of two different light fluxes, $A$ and $B$. Flux $A$ passes through the web twice to measure its thickness. Flux $B$ directly enters the photo tube. A semicircular shutter making 70 revolutions per minute allows the photo tube to receive light flux $A$ and light flux $B$ alternately, so that in no case do $A$ and $B$ go into the photo tube simultaneously. The semicircular shutter also controls electrical switches so that the photo tube is connected to the amplifier assigned exclusively for the measurement of web thickness when the tube is receiving light flux $A$ and that the tube is connected to the electronic servo which keeps the intensity of light source constant when the tube is receiving flux $B$. Part of the electronic circuits used in the measuring element is shown in Fig. 2.

The control equipment was mounted on the last card of a three-card woolen carding set (Fig. 1(a)).

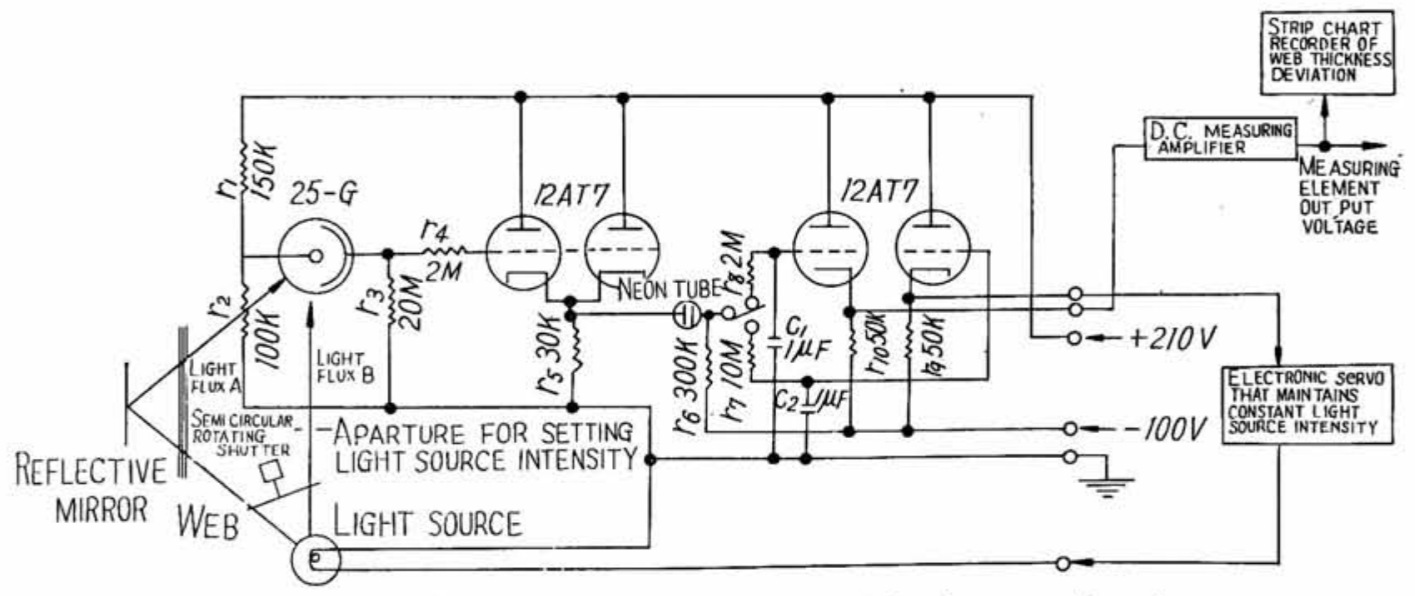

Fig. 2 Initial stages of electronic circuit used in the measuring element 
The card had a doffer diameter of $1,500 \mathrm{~mm}$ ( $58 \mathrm{in}$ ) and an average doffer speed of 3.0 r.p.m. (or 13.3 $\mathrm{m} / \mathrm{min}$ in average delivery speed).

\section{Procedures of Experiments and Analysis of Data}

First, the dynamic characterics of the control equipment was measured by a number of step response tests and an oscillation test. Then the statistical properties of the yarn weight variation and those of the random noise generated in the measuring element were computed with the aid of an analog type correlation analyser. Finally, by combining the statistical properties of yarn weight signals and the dynamic characteristic of the control equipment obtained previously, the authors computed theoretically the extent by which the yarn weight variation could be reduced by the control equipment. The theoretical prediction on yarn weight variation under automatic control thus obtained agreed fairly well with the experimental values. The adequacy of the analytical methods used was, therefore, well proved.

\section{Determining the Dynamic Characteristics of the Control Equipment}

(1) For convenience of experiments and analysis, the control system was divided into four component elements: the measuring element, the controlling element, the manipulating element and the card. Figs. 1(a) and 1(b) show the four component elements and the signals between them.

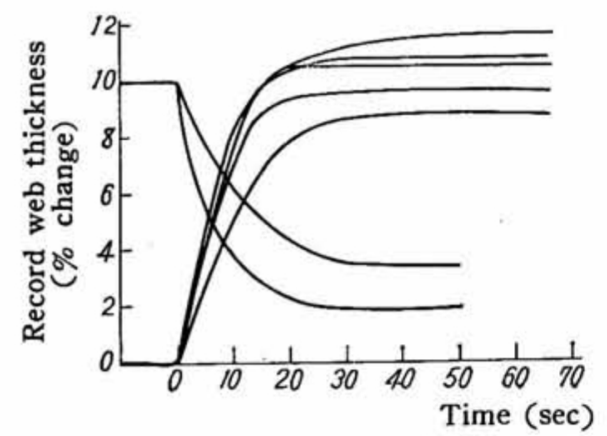

Fig. 3 Changes in recorded web thickness in response to sudden insertion of a cellophane sheet under the measuring element
(2) In measuring the dynamic characteristics of the measuring element, the card was stopped with a $14 \mathrm{~s}$ web placed under the measuring element. Then a sheet of cellophane was placed abruptly on top of the web under the measuring element. The resulting change in the recorded web thickness on the strip-chart recorder is as shown in Fig. 3.

Note here that the strip-chart recorder is so rapid in response that the time lag of 10 seconds observed in Fig. 3 is solely the time lag of the measuring element, not that of the strip chart recorder.

Fig. 4 is the calibration of strip-chart recorder with respect to web thickness in terms of the equivallent roving count. The figure, however, does not show the proportional gain of the measuring element, because it relates the web thickness to stripchart indication but not to the output voltage of the measuring element. The proportional gain of the measuring element, however, is immaterial here because what is needed in later analysis is the overall gain of all component elements (loop gain) which can be measured later by the oscillation test.

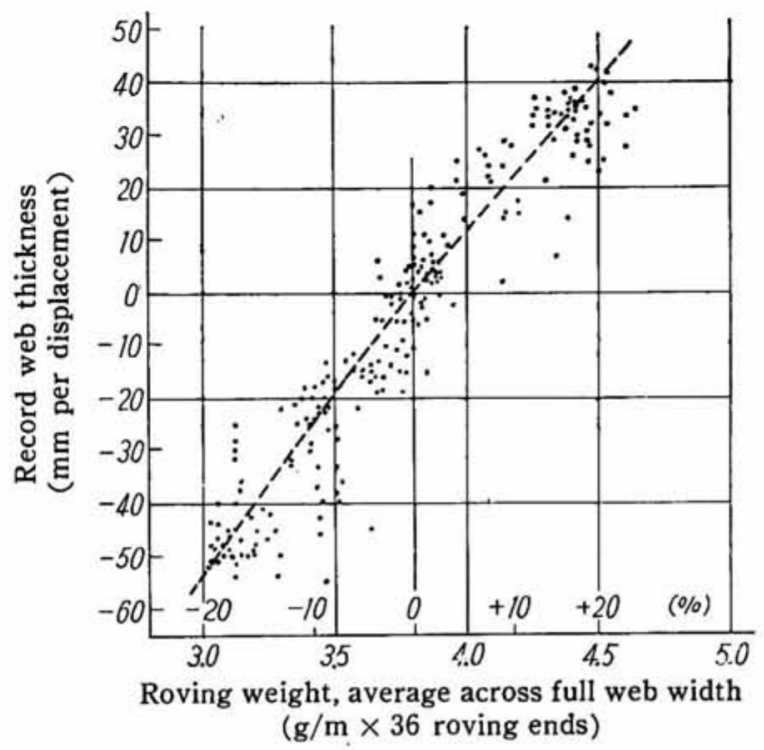

Fig. 4 Static characteristics of measuring element

Denote the proportional gain $K_{1}$ and recall that the dynamic characteristics of the measuring element are approximated by a time constant of $10 \mathrm{sec}$ as observed in Fig. 3. The transfer function for the measuring element, then, is

$$
G_{M}(s)=\frac{K_{1}}{1+10 s}
$$


(3) Dynamic characteristics of the controlling element

The nature of the electronic circuits used in the controlling element made it difficult to measure the input and output voltages without imparting undesirable disturbances to the control system.

Therefore, the assumption was made that the controlling element had the dynamic characteristics as expressed by the transfer function

$$
G_{c}(s)=\frac{R_{f}}{R_{\imath}}\left(1+\frac{1}{C_{f} R_{f} s}\right)
$$

which was given in an instruction mannual supplied by the manufacturer of the controlling element. $R_{l}, R_{f}$ and $C_{f}$ in eq. (2) are resistance and capacitance used in the circuit shown on the left-hand side of Fig. 1(a). $\quad C_{f}$ has a fixed value of $8 \mu f . \quad R_{\iota}$ and $R_{f}$ can be set to any values within the range of $R_{i}=0 \sim 12 \mathrm{M} \Omega$ and $R_{f}=0.2 \sim 2.0 \mathrm{M} \Omega$. During the experiments under review, however, $R_{f}$ was always fixed to the value $R_{f}=1.2 \mathrm{M} \Omega$. Eq. (2), therefore, reduces itself to

$$
G_{o}(s)=\frac{1}{R_{\ell}}=\frac{1+9.6 s}{8 s}
$$

(4) Dynamic characteristics of the manipulating element

In the manipulating element the authors included the variable speed drive and the electrical servo. The manipulating element can either be driven automatically by the output voltage of the controlling element or manually by a potentiometer.

To determined the dynamic characteristics, the manipulating element was put to mannual control and an abrupt stepwise change was given to the potentiometer setting. Changes in the clearer roll speed (which is exactly in proportion to the doffer speed) that follows the abrupt changes in the potentiometer setting are shown in Figs. 5(a) and 5(b). These test results indicate that the time constant depends both on the magnitude of the stepwise input signal given and on the direction of the stepwise change, as shown in Fig. 6.

The reason why the magnitude of the input signal affects the time constant is that the speed of the two-phase motor saturates on receiving large input signals. The effect of the direction of the input signal can be explained by the fact that it takes a larger torque to turn the control shaft of the variable speed drive in the direction of a decreasing doffer speed than in the opposite direction.
Since normal operating conditions of the manipulating element correspond to the lower left region of Fig. 6, the dynamic characteristics of the manipulating element was approximated by a first order lag with a time constant of 8 seconds.

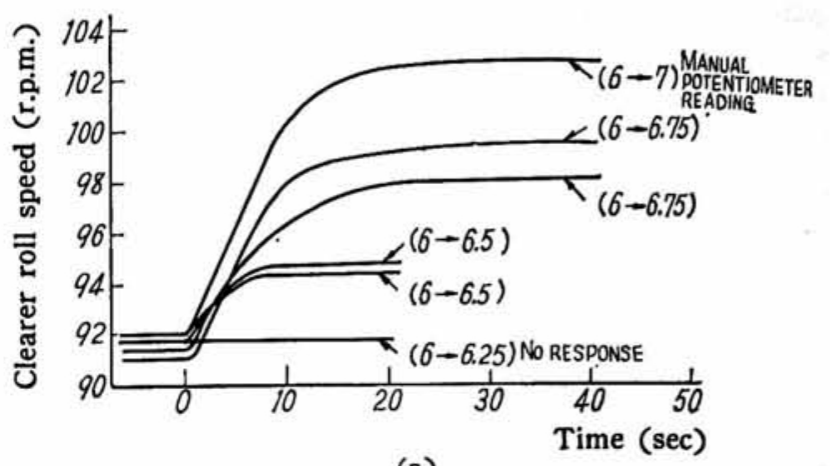

(a)

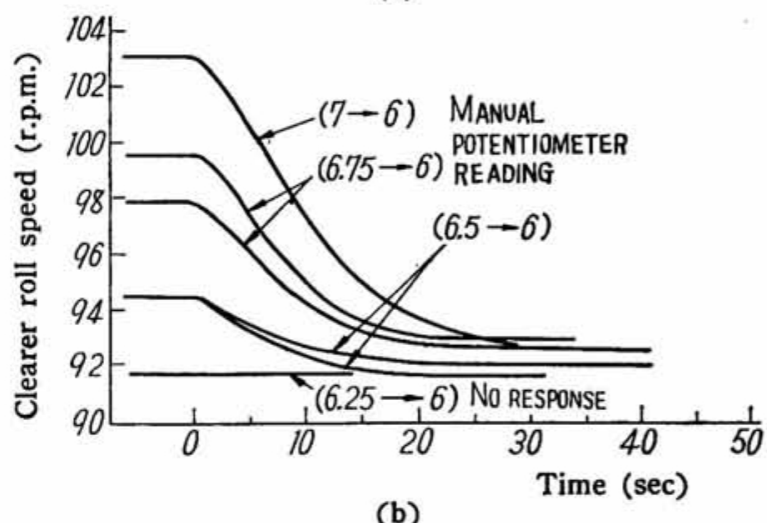

(Clearer roll speed is exactly proportional to doffer speed)

Fig. 5 Step responses of manipulating element

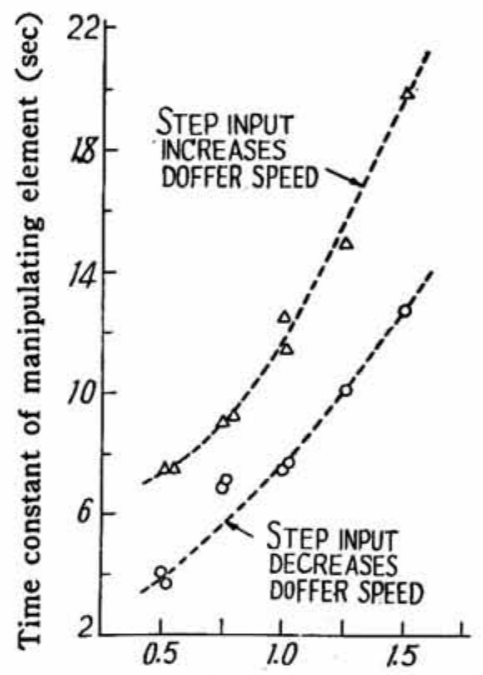

Magnitude of step input (in potentiometer dial reading)

Fig. 6 Effect of direction and magnitude of input signal on the time constant of manipulating element 


$$
G_{A}(s)=\frac{K_{3}}{1+8 s}
$$

(5) Characteristics of the card

To insure accuracy, the characteristics of the card were tested doubly by using two different test methods: the step response test and the oscillation test. A good agreement was observed between the results of the two independent tests (see Figs. 7 (a) and 7 (b)).

The wavy solid curve in Fig. 7 (a) shows the changes in the recorded web thickness on the stripchart recorder in response to a ramp-shaped change in the doffer speed (indicated in terms of clearer roll speed) as shown in the same figure. A truely stepwise change in the doffer speed was not possible because of the saturation in the servo speed as previously mentioned. A ramp-shaped input, however, is perfectly satisfactory as a substitute for a stepwise input signal although it slightly complicates calculation.

The dotted curve in Fig. 7 (a) is a computed curve, derived on the assumption that the card has the characteristics of eqs. (6) and (12) as obtained in the oscillation test; and that a card with such

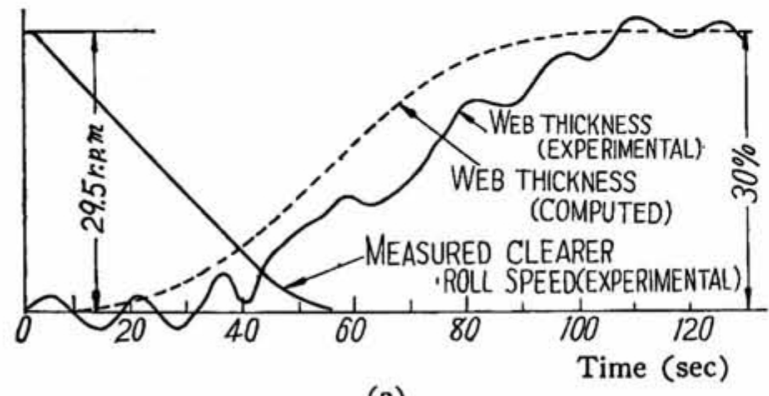

(a)

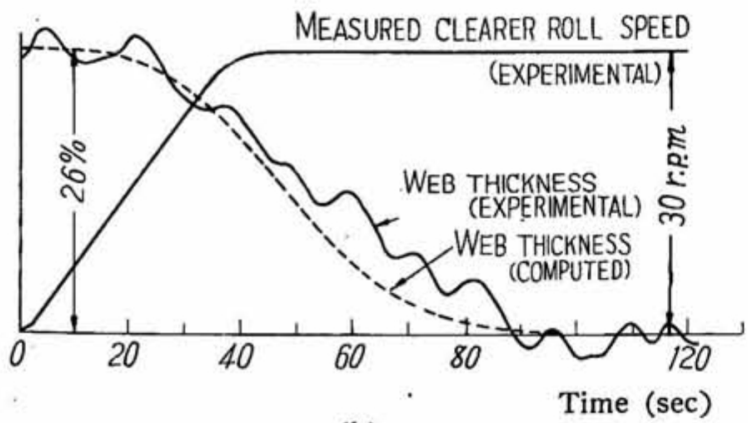

(b)

Computed values of web thickness is the response of the transfer function $\frac{-e^{-12 S}}{(1+12 s)(1+10 s)}$ to the rampshaped input shown above as clearer roll speed

Fig. 7 Transient response of the card, experimental values and computed values characteristics was subject to the ramp-shaped input shown in Fig. 7(a).

The proximity of the dotted curve to the wavy solid curve in Fig. 7(a) means good agreement between the results of the transient response test and those of the oscillation test. Fig. 7 (b) is a mere repetition of $7(a)$, except for the direction of the input signal.

Fig. 8 shows the static characteristic of the card and indicates that, in the long run, the roving count is in direct proportion to the doffer speed (take-up roll speed in Fig. 8 is exactly proportional to the doffer speed). In other words, a $1 \%$ increase in the doffer speed reduced the web thickness by $1 \%$ in the long run if there are no noise inputs. Consequently the proportional gain $K_{3}$ of the card is

$$
K_{3}=-1\left(\frac{\% \text { change in web thickness }}{\% \text { change in doffer speed }}\right)
$$

although $K_{3}$ is immaterial in the statistical analysis which will be discussed later, because it is only the loop gain, not the proportional gains of individual elements, that counts.

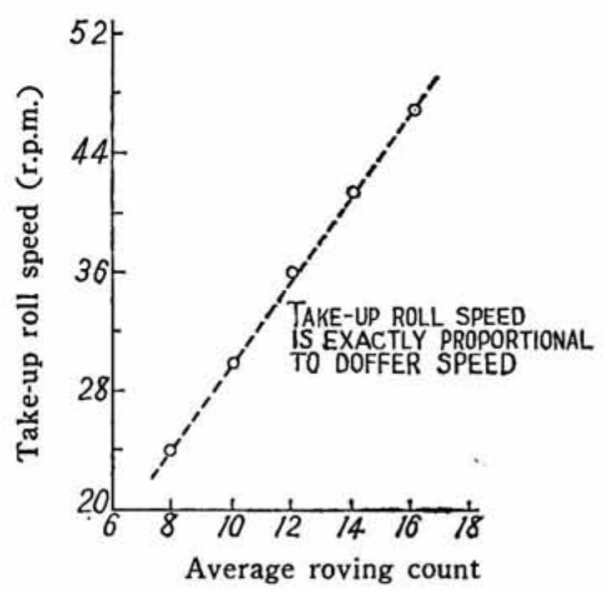

Fig. 8 Static characteristic of card

The transfer function of the card is discussed in the next section in relation to the oscillation test.

(6) Oscillation test

The oscillation test was made to determine the loop gain of the control system and the major time constant $T$ of the card. The loop gain is the product of the proportional gains of all the component elements in the control system.

It was first assumed that the card had a transfer function of the shape

$$
G_{p}(s)=\frac{K_{3} e^{-L s}}{1+T s}
$$




\section{April 1963}

$e^{-L S}$ in the numerator stands for the transportation time the web takes to travel from the cylinder-doffer contact where the draft change occurs to the measuring element of the control equipment (see Fig. $1(\mathrm{a}))$.

$L$ is known to be

$$
L=12 \mathrm{sec}
$$

$K_{3}$ is $K_{3}=-1$ as stated in the preceding section. Eq. (5) therefore, reduces itself to:

$$
G_{p}(s)=\frac{-e^{-12 s}}{1+T s}
$$

The card then was run with the control equipment set to "Automatic" position, and the resistance $R_{t}$ in the controlling element was reduced abruptly from the initial $R_{t}=1.2 \mathrm{M} \Omega$ to $R_{t}=0.8 \mathrm{M} \Omega$. This made the control system unstable and produced a diverging oscillation as shown by the web thickness record (solid curve) in Fig. 9.

The period of the oscillation increased as its amplitude grew because of the speed saturation in the manipulating element. It was estimated that $130 \mathrm{sec}$ was the period of oscillation which corresponded to the amplitude experienced in normal operations. Major time constant $T$ of the card was obtained as explained below.

Loop transfer function $F(s)$ of the control system is the product of the transfer functions of all the component elements. By multiplying eqs. (1), (3), and (6) we obtain $F(s)$ as

$$
\begin{aligned}
F(s) & =\frac{K_{1}}{1+10 s} \frac{1+8 R_{f} s}{8 R_{t} s} \frac{K_{2}}{1+8 s} \frac{e^{-12 s}}{1+T s} \\
& =\frac{K_{1} K_{2}(1+9.6 j \omega) e^{-12 j \omega}}{8 R_{t} j \omega(1+10 j \omega)(1+8 j \omega)(1+T j \omega)}
\end{aligned}
$$

where $s=j \omega$ and $R_{f}=1.2$

The value of $\omega$ corresponding to the above mentioned period $130 \mathrm{sec}$ is

$$
\omega=\frac{2 \pi}{130}=0.0482 \mathrm{rad} / \mathrm{sec}
$$

For the card to oscillate with a period of $130 \mathrm{sec}$. the following relation must be established:

$$
\angle F(\omega=0.0482)=-180^{\circ}
$$

From eqs. (7) and (9) we obtain

$$
\begin{aligned}
& \tan ^{-1}(9.6 \times 0.0482)-\frac{180^{\circ}}{\pi} \times 12 \times 0.0482 \\
& -\tan ^{-1}(10 \times 0.0482)-\tan ^{-1}(8 \times 0.0482) \\
& -\tan ^{-1}(0.0482 T)-90^{\circ}=-180^{\circ}
\end{aligned}
$$

By solving eq. (10) for $T$ we get

$$
T=12.0 \mathrm{sec}
$$

The transfer function $G_{p}(s)$ of the card is now fully determined as

$$
G_{p}(s)=\frac{-e^{-12.0 s}}{1+12.0 s}
$$

The dotted lines in Fig. 7(a) are the response of $G_{p}(s) \times G_{M}(s)$ to the ramp-shaped input signal shown in Fig. 7 (a).

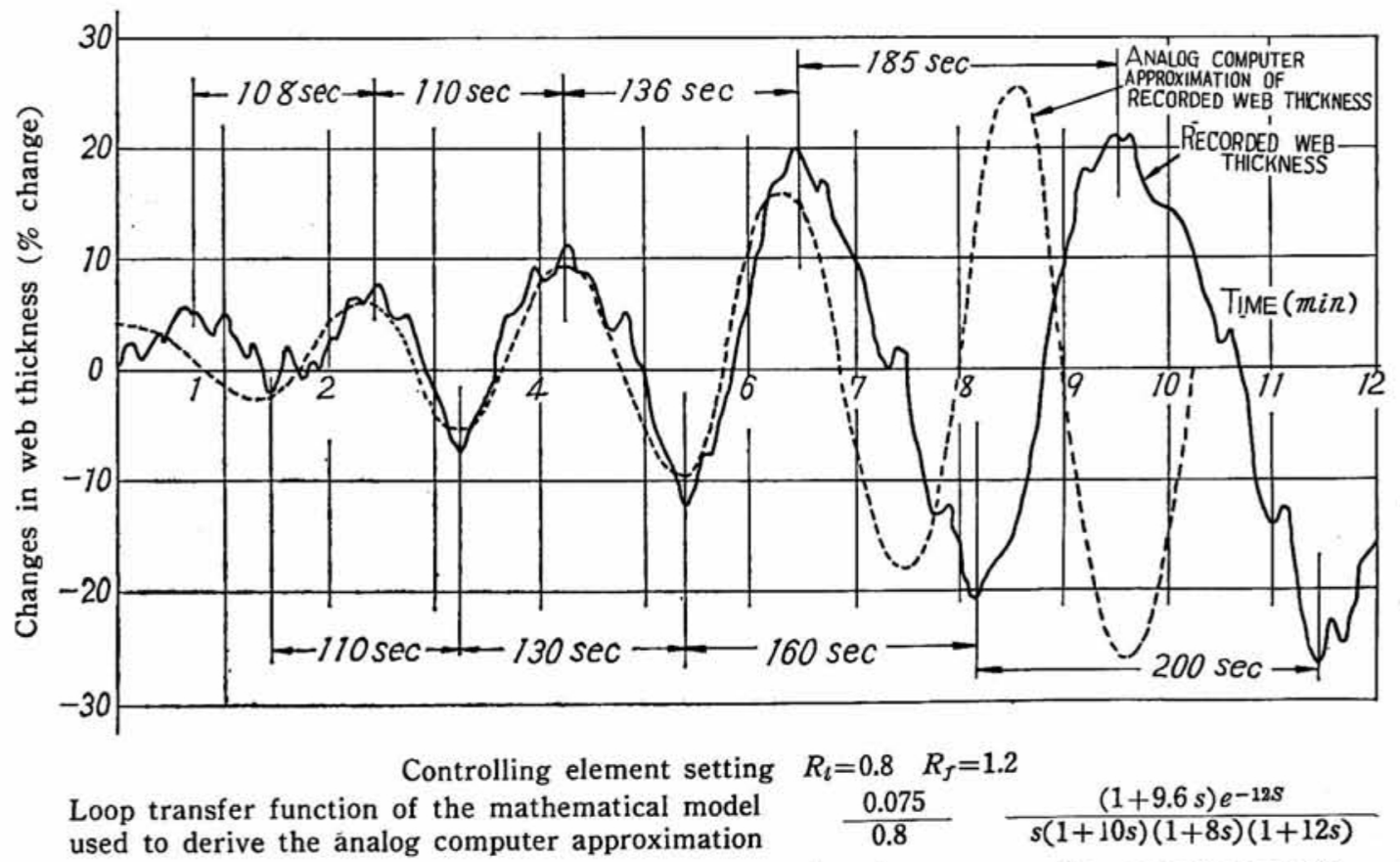

Fig. 9 Oscillation test of the card and its approximation on on analog computer circuit 
As explained before, it agrees quite well with the actual transient response of the web thickness obtained in field tests and shown in a solid wavy curve in Fig. 7 (a). This result proves the validity of the oscillation test.

Now we proceed to the second objective of the oscillation test, namely, the determination of the loop gain.

By substituting all the information obtained so far on the system characteristics into the block diagram in Fig. 1(b), the block diagram shown in Fig. 10 is obtained. $R_{l}$ and $R_{f}$ in Fig. 10 are known values which can be read directly off the potentiometer dials on the controlling element. $\frac{K_{1} K_{2}}{8}$ is the loop gain whose numerical value is left unknown at this stage of analysis.

To determine the value of $\frac{K_{1} K_{2}}{8}$, the block diagram in Fig. 10 is first converted into analog computer circuit, then the loop gain of the computer circuit is gradually increased until the computer output exhibits a diverging oscillation, most similar in shape to that of the card shown in Fig. 9. Since the loop gain of the computer circuit is then equal to that of the card, it is read off the computer as the estimated value of $\frac{K_{1} K_{2}}{8}$.

The loop transfer function given in Fig. 10 is first simplified before it is simulated on the analog computer. $(1+10 s)$ and $(1+9.6 s)$ in the denominator and numerator of the loop transfer function are so similar that the two factors are considered to cancel each other.

$$
\begin{aligned}
F(s) & =\frac{K_{1} K_{2}}{8 R_{t}} \frac{(1+9.6 s) e^{-12 s}}{S(1+10 s)(1+8 s)(1+12 s)} \\
& \doteqdot \frac{K_{1} K_{2}}{8 R_{t}} \frac{e^{-12 s}}{s(1+8 s)(1+12 s)}
\end{aligned}
$$

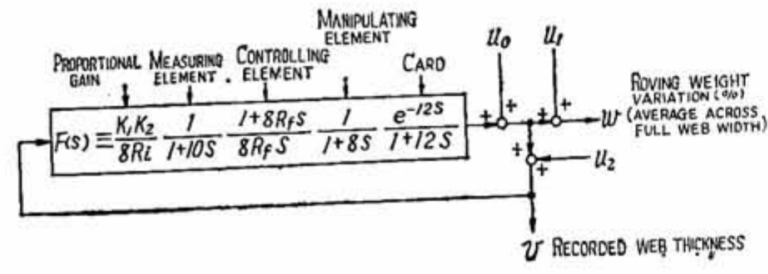

$\left(\begin{array}{l}\text { Strictly speaking } \frac{1}{1+10 s} \text { should be placed be- } \\ \text { tween } v \text { and } v_{2} \text {, but above simplification does not } \\ \text { change the picture materially. }\end{array}\right)$ (See Fig. 16 for $u_{0}, u_{1}$ and $u_{2}$ )

Fig. 10 Block diagram for woolen card control system
Time constants as they appear in eq.(13) are too large to be simulated on the analog computer. Therefore, the time scale was changed by a factor of $2 / 9$ by substituting $s$ with $2 / 9 s^{\prime}$.

$$
s=2 / 9 s^{\prime}
$$

Substituting eq.(14) into eq.(13) gives us

$$
F(s)=\frac{K_{1} K_{2}}{8 R_{t}}-\frac{9}{2} \frac{e^{-2.60 s}}{s^{\prime}(1+1.78 s)(1+2.66 s)}
$$

It is not easy to simulate the factor $e^{-2.068^{\prime}}$ on a computer circuit. Accordingly, the last three factors in eq.(15) were approximated by the fourth power of a first-order lag having a time constant of $T^{\prime}$.

$$
\begin{aligned}
F(s) & =\frac{K_{1} K_{2}}{8 R_{\imath}} \frac{9}{2} \frac{e^{-2.6 B S^{\prime}}}{s\left(1+1.78 s^{\prime}\right)\left(1+2.66 s^{\prime}\right)} \\
& \doteqdot \frac{A}{s^{\prime}\left(1+T^{\prime} s\right)^{4}}
\end{aligned}
$$

where

$$
A \equiv \frac{K_{1} K_{2}}{8 R_{i}} \frac{9}{2}
$$

The value of $T^{\prime}$ was chosen in such a way that the sum total of time constants on the right-hand side of eq.(16) was equal to that on the left-hand side.

That is to say,

$$
\begin{aligned}
& 2.66+1.78+2.66=4 T^{\prime} \\
& \text { or } T^{\prime}=1.78 \mathrm{sec}
\end{aligned}
$$

The left-hand side and the right-hand side of eq. (16) are plotted as two vector diagrams in Fig. 11. The proximity of these two vector diagrams proves that the approximation used in eq.(16) is highly accurate. Gain constant $A=0.115$ used in Fig. 11 is an arbitrary value.

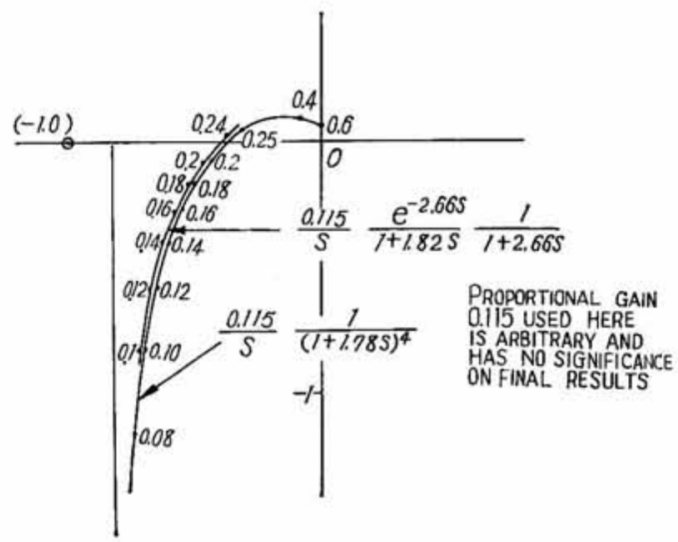

Fig. 11 Comparison of loop transfer function and its approximation on vector diagram

Docernal of The 7exule Machinery Society of Dapain 
The block diagram for the card shown in Fig. 10 is now approximated by the block diagram in Fig. 12.

The computer circuit which corresponds to the block diagram in Fig. 10 is shown in Fig. 13 where $P$ is the dial indication on the potentiometer No. 9 . $P$ should, of course, be proportional to $A$ and the proportionality constant between them is determined by a calibration procedure as described below.

Fig. 11 shows that $A=0.320$ should make the system critically damped. The computer circuit of Fig. 13 shows a critically damped state when $P$ is set to 0.031 as shown in Fig. 14 (a). Therefore, the proportionality constant between $P$ and $A$ is

$$
\frac{P}{A}=\frac{0.031}{0.32}=0.097
$$

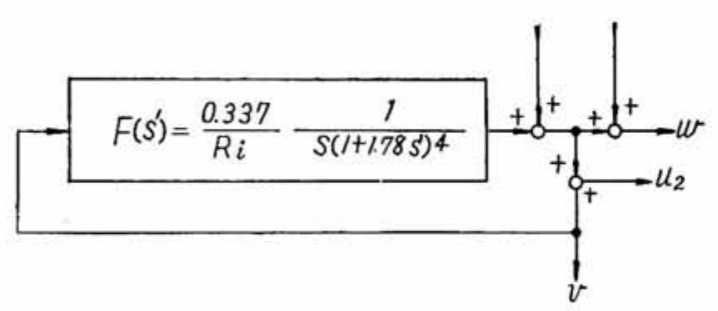

$R_{f}=1.2$

Laplace transform equation corresponding to the above flock diagram is

$$
W=V_{1}+\frac{1}{1+F} V_{0}+\frac{F}{1+F} V_{2}
$$

Fig. 12 Block diagram for woolen card control system as expressed in computer time

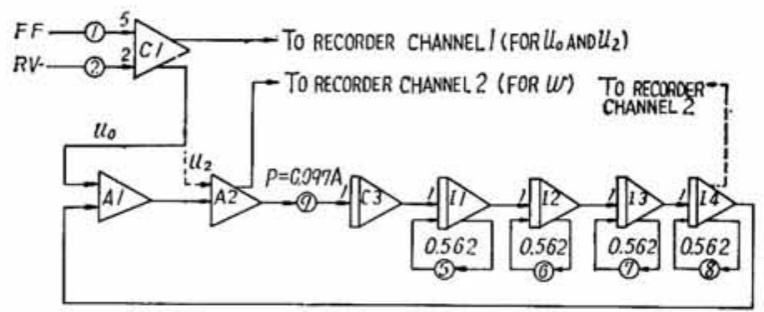

Fig. 13 Analog computer circuit for the block diagram in Fig. 12

When $P$ is increased to the value $P=0.041$, the computer circuit exhibits a diverging oscillation test whose rate of divergence matches most closely that of the solid curve in Fig. 9. The diverging oscillation thus obtained on the analog computer is shown in Fig. 14(b). The dotted line in Fig. 9 is identical to the one shown in Fig. 14(b), except that the time scale is re-converted into real time by multiplying the afore mentioned factor $2 / 9$.

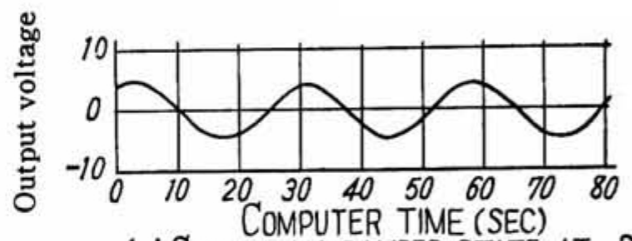

(a) CRITICALLY DAMPED STATE AT $P=0.031$

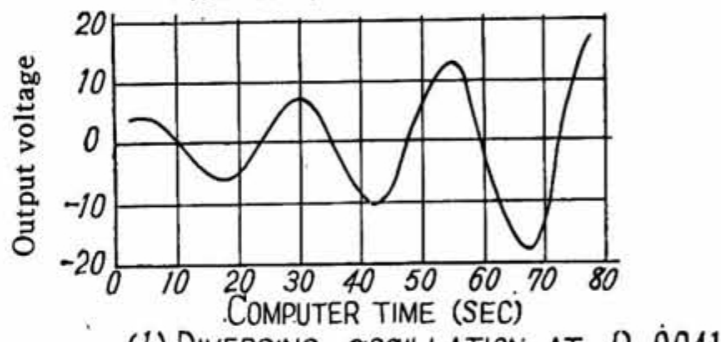

(b) DIVERGING OSCILLATION AT $P=0.041$

Fig. 14 Analog computer output

Now that the diverging oscillation of the card control system is reproduced on a computer circuit, and considering that the value of $R_{t}$ used in the oscillation test is $R_{t}=0.8 \mathrm{M} \Omega$, the loop gain $\frac{K_{1} K_{2}}{8}$ in eq.(13) is determinable by substituting $P=0.041$ and $R_{\ell}=0.8 \mathrm{M} \Omega$ in eqs. (17) and (19).

Thus:

$$
A=\frac{P}{0.097}=\frac{0.041}{0.097}=\frac{K_{1} K_{2} \times 9}{8 R_{\imath}} \frac{9}{2}=\frac{K_{1} K_{2} \times 9}{8 \times 0.8 \times 2}
$$

Therefore,

$$
\frac{K_{1} K_{3}}{8}=\frac{0.041 \times 2 \times 0.8}{0.097 \times 9}=0.075
$$

By substituting eqs.(11) and (20) into eq.(7), the loop transfer function is fully obtained as

$$
F(s)=\frac{0.075\left(1+8 R_{f} s\right) e^{-12 s}}{R_{t} s(1+10 s)(1+8 s)(1+12 s)}
$$

where $R_{t}$ and $R_{f}$ are the potentiometer setting of the controlling element and are changeable over the range $R_{\imath}=0 \sim 12 \mathrm{M} \Omega$ and $R_{f}=0.2 \sim 2.0 \mathrm{M} \Omega$.

\section{Analyzing Process Variables by Statistical Methods}

The preceding chapters described the dynamic characteristic of the card control system by a mathematical model consisting of several transfer functions. Such a mathematical model, however, is not useful unless it can be related directly to the actually measured values of roving weight variation.

Statistical methods, therefore, are used in this chapter on the various process variables to relate 
the mathematical model to the numerically expressed values of roving weight variation. Roving weight variation is usually expressed in terms of the coefficient of variation (C.V.\%) of the weight of consecutively cut roving somples, or the spectrum density, or the C.V. versus cut length curve. In the present study the first three expressions of roving weight variation are discussed in relation to the mathematical model obtained in chapter 4 .

Another reason for the use of statistical methods is the presence of ramdom noise in the control system. There are three major noise sources in this control system, all of them have such a mag. nitude that the omission of any of them would result in a grave inaccuracy of the analysis. The statistical methods are most useful for the analysis of such random noise.

The three different noise sources are denoted as $u_{0}, u_{1}$ in Figs. $1(\mathrm{~b}), 10,11$, and 16. $u_{0}$ is the nonuniformity in the weight of the wool fleece supplied to the card. The primary object of the control equipment is course, to eliminate the effect of $u_{0}$ on roving weight. As shown in Fig. 15, the full width of web is divided by the tape condenser into 144 parallel roving ends. Since the card control equipment is intended to reduce the roving weight variation in a lengthwise direction and does not touch upon the weight variation across the card web we should take the average weight of 144 roving ends as the controlled variable. However, the difficulty in combing 144 ends into one bundle made it necessary to use only half of them as roving samples. $u_{1}$ is a ramdom noise caused by this sampling.

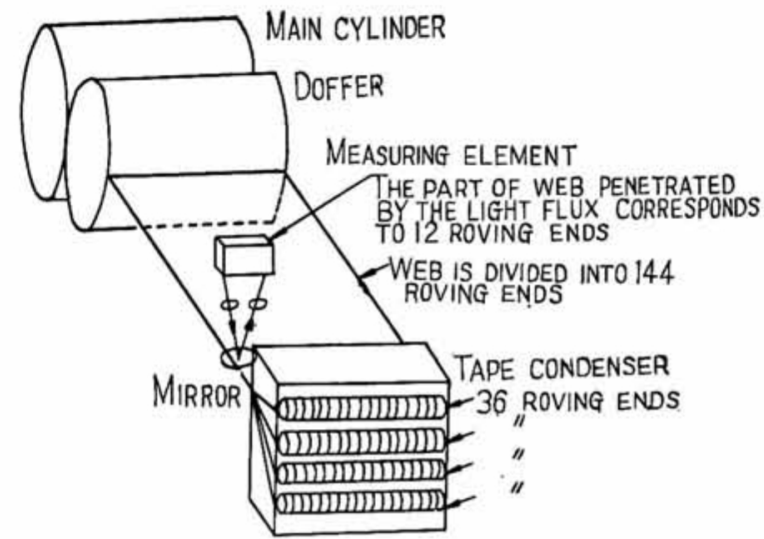

Fig. 15 Location of measuring element

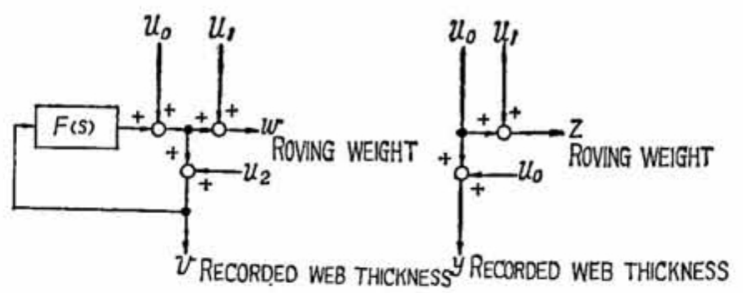

(a) Card under automatic control

(b) Automatic control cut off

$u_{0}$ : Random noise caused by the variation in the weight of wool fleece supplied to the card

$u_{1}$ : Random noise caused by the sampling of 64 roving ends out of the total of 144 ends in measuring roving weight

$v_{2}$ : Random noise caused because the measuring element covers only $8 \%$ of the web width. Electronic noise in the measuring element is also included in $u_{2}$.

Fig. 16 Noise inputs entering the control system

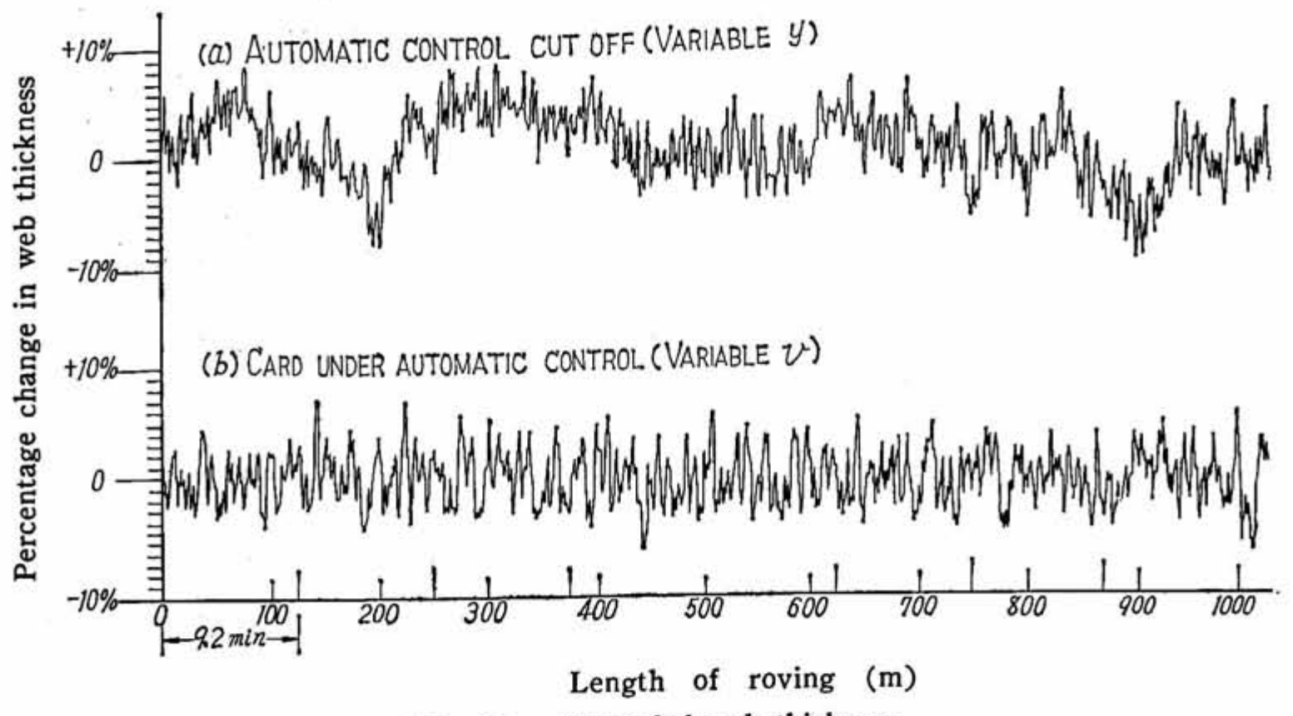

Fig. 17 Recorded web thickness 
$u_{2}$ is the noise created during the measurement of web thickness. A major part of $u_{2}$ is the second sampling noise which is created because the measuring element of the control equipment covers only $8 \%$ of the width of the web as shown in Fig. 15. The rest of $u_{3}$ is the electronic noise generated in the measuring element and is impertinent to the web thickness.

$u_{0}, u_{1}$ and $u_{2}$ are related to the roving weight and the strip-chart indication as shown in Fig. 16. $w$ is the weight of consecutively cut two meter roving samples, each with 64 roving ends. $v$ is the web thickness as indicated on the strip-chart recorder. The symbols $w$ and $v$ are used for a case where the control equipment is in automatic run. The same variables are denoted $z$ and $y$ for a case where the control equipment is in manual run. Fig. 17 shows variables $y$ and $v$ as recorded on the stripchart.

The yarn weight $z$ and the recorded web thickness $y$ can be measured easily, the three noise sources, $u_{0}, u_{1}$ and $u_{2}$, in the form of time function do not render themselves to experimental measurements. However, it is possible to derive the statistical properties of $u_{0}, u_{1}$ and $u_{3}$ out of the measured values of $y$ and $z$. Their derivation is as follows: The autocorrelation $\varphi u_{0} u_{0}(\tau)$ of a time function $u_{0}$ is defined as

$$
\varphi u_{0} u_{0}(\tau)=\lim _{r \rightarrow \infty} \frac{1}{2 T} \int_{-\tau}^{\tau} u_{0}(t) u_{0}(t+\tau) d t
$$

Since $u_{0}, u_{1}$ and $u_{2}$ can be assumed to be statistically independent these variables and their auto. correlations have the relations as shown in eqs. (23) to (27).

$$
\begin{gathered}
u_{0}+u_{1}=z \\
u_{0}+u_{2}=y \\
\varphi_{u 0 u 0}+\varphi_{u 1 u s=}=\varphi_{z z} \\
\varphi_{u 0 u 0}+\varphi_{u s u 2=}=\varphi_{v v}
\end{gathered}
$$$$
\varphi_{v-z}, v-z \varphi_{u=2-u 1}, u 8-u 1=\varphi_{u s u s}+\varphi_{u 1 u 1}
$$
we get

Solving eqs. (25), (26) (27) for $\varphi_{u s u s}$ and $\varphi_{u 1 u 1,}$

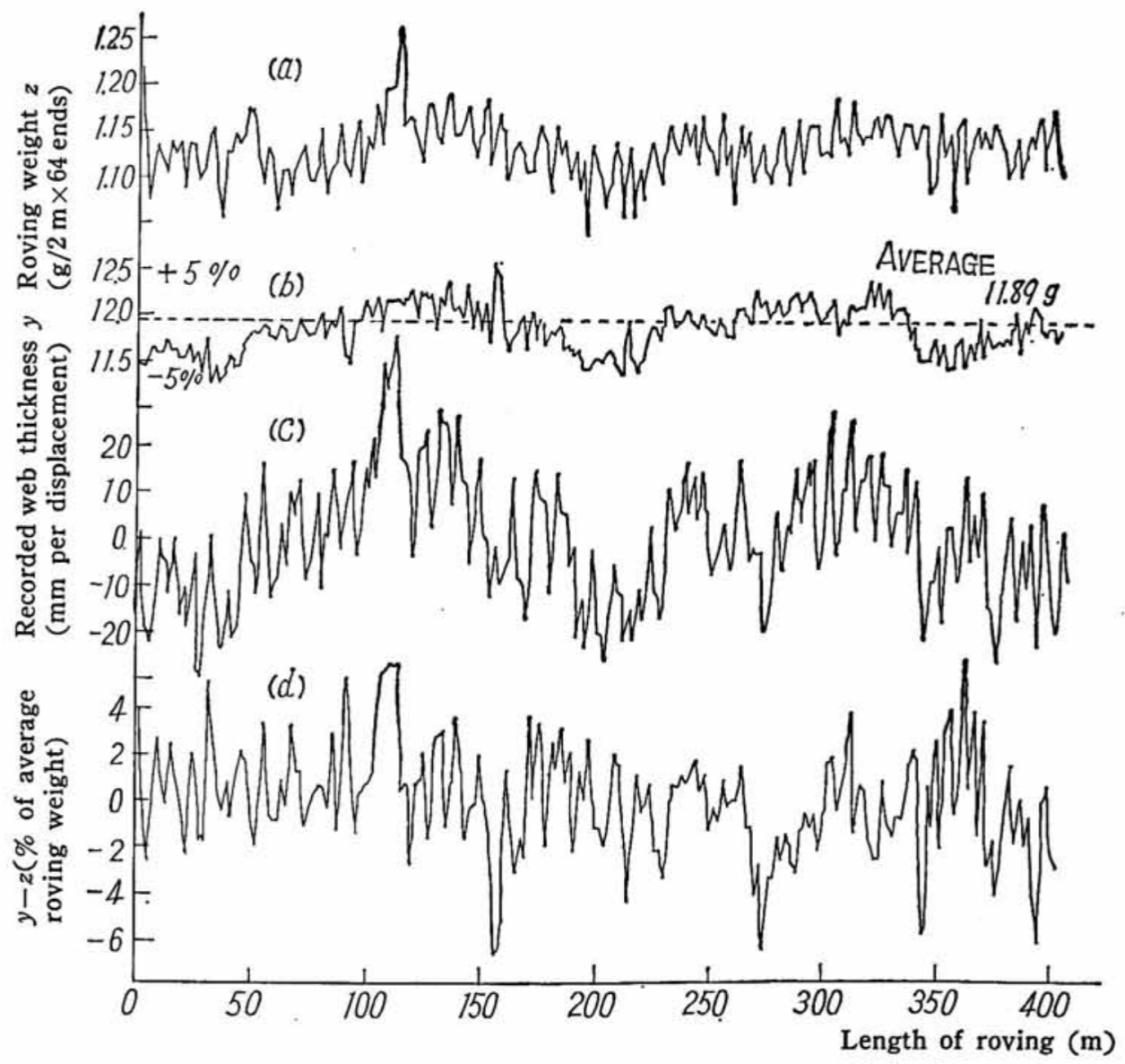

Fig. 18 Recorded web thickness and roving weight measured simultaneously 


$$
\begin{aligned}
& \varphi_{u \Omega u 2}=\frac{1}{2}\left(\varphi_{v v}-\varphi_{z z}+\varphi_{\nu-z}, v-z\right) \\
& \varphi_{u 1 u 1}=\frac{1}{2}\left(-\varphi_{v y}+\varphi_{z z}+\varphi_{\nu-v, v-z}\right)
\end{aligned}
$$

$\varphi_{v y}, \varphi_{z z}$ and $\varphi_{y-z}, y-z$ are derived from the measured values of $y$ and $z$ by using eq.(24). The actual computation of $\varphi_{v v}, \varphi_{z z}$ and $\varphi_{v-z}, v-z$ is made on a analog type correlation analyser which is made exclusively for the computation of eq. (24).

Autocorrelations $\varphi_{v v}, \varphi_{z z}, \varphi_{y-z} v-z, \varphi_{u \Omega}$, and $\varphi_{u 1 u 1}$ thus computed are shown in Fig. 20.

Curves (b) and (c) in Fig. 18 are, respectively $z$ and $y$ which are measured experimentally. In Fig. 18, however, $y$ is plotted in milimeter displacement, while $z$ is plotted in gramms of roving weight. In Fig. 19 the corresponding values of $y$ and $z$ are plotted to determine the conversion factor between the units of $y$ and $z$. Fig. 19 is similar to Fig. 4, except for a shift in the origin.

By using the conversion factor obtained from the regression line in Fig. 19, $y$ and $z$ are graduated in percentage deviation from the mean value to compute their difference $y-z$. Curve (a) in Fig. 18 is shown as a reference and is the weight of six roving ends which correspond to the part of the web penerated by the light flux from the measuring element.

Curve (a) shows a better correlation to the recorded web thickness $y$ than does curve (b), but the fact that (a) still is not identical to $y$ indicates the presence of an electronic noise in the measuring element.

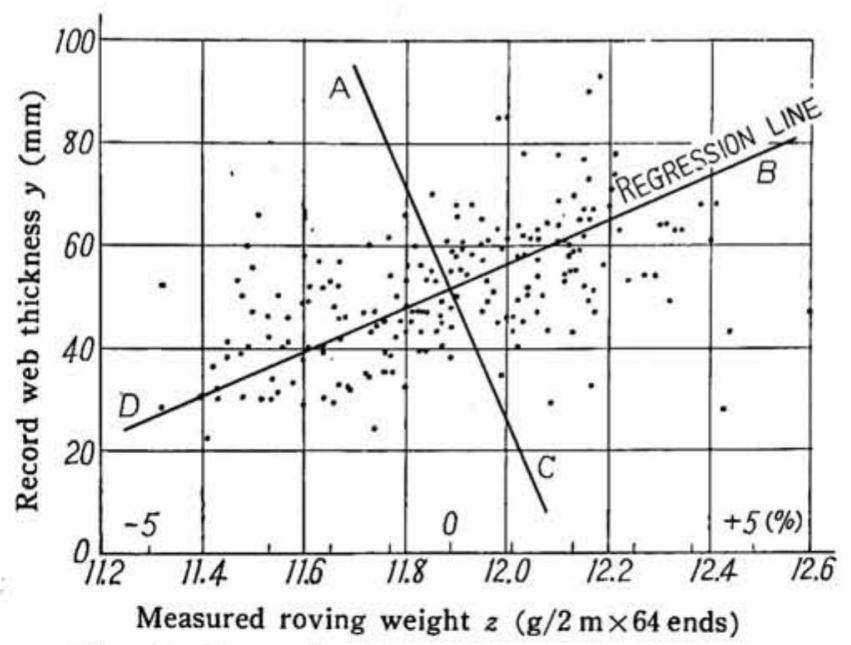

Fig. 19 Regression diagram for variables $y$ and $z$
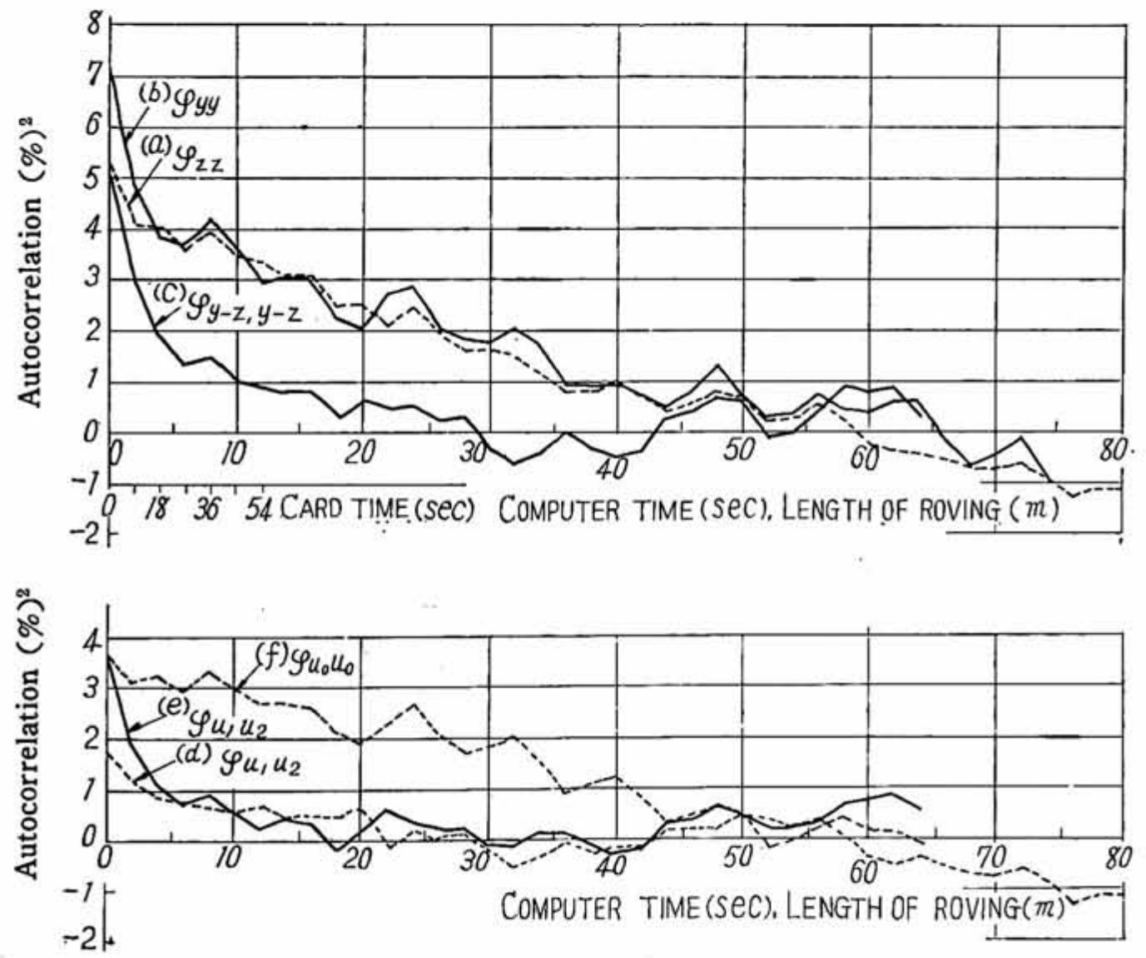

Fig. 20 Autocorrelation of input and output variables 
Now that the statistical properties of various input signals are expressed in terms of autocorrelations, we proceed to fit them into the mathematical model obtained in the previous chapters. For this purpose, the autocorrelations obtained must be converted into the spectrum density $\emptyset(s)$. The spectrum density $\emptyset_{u 1 u 1}(s)$ of an autocorrelation $\varphi_{u 1 u 1}(\tau)$ is defined by either eq. (30) or eq.(31).

$$
\begin{gathered}
\int_{-\infty}^{\infty} \varphi_{u 1 u 1}(\tau) e^{-s \tau} d \tau=\emptyset_{u 1 u 1}(s) \\
\frac{1}{2 \pi j} \int_{-j \infty}^{j_{\infty}} \emptyset_{u 1 u 1}(s) e^{\delta \tau} u \tau=\varphi_{u 1 u 1}(\tau)
\end{gathered}
$$

When the variables $u_{0}, u_{1} u_{2} v$ and $w$ are mutually related as shown in the block diagram of Fig. 16(a) the spectrum density of these variables satisfies eq. (32).

$$
\emptyset_{w w}=\emptyset_{u 1 u 1}+\left|\frac{1}{1+F(S)}\right|^{2} \emptyset_{u 0 u 0}+\left|\frac{F}{1+F(S)}\right|^{2} \emptyset_{u 3 u 3}
$$

where $F\left(s^{\prime}\right)$ is the loop transfer fuction of the control system given in eq. (16). Note here that the time scale in eq.(32) is computer time, rather than real time.

To compute their spectrum density, $\varphi_{u 2 u s}$ and $\varphi_{u 0 u}$ shown in Fig. 20 are approximated by simple

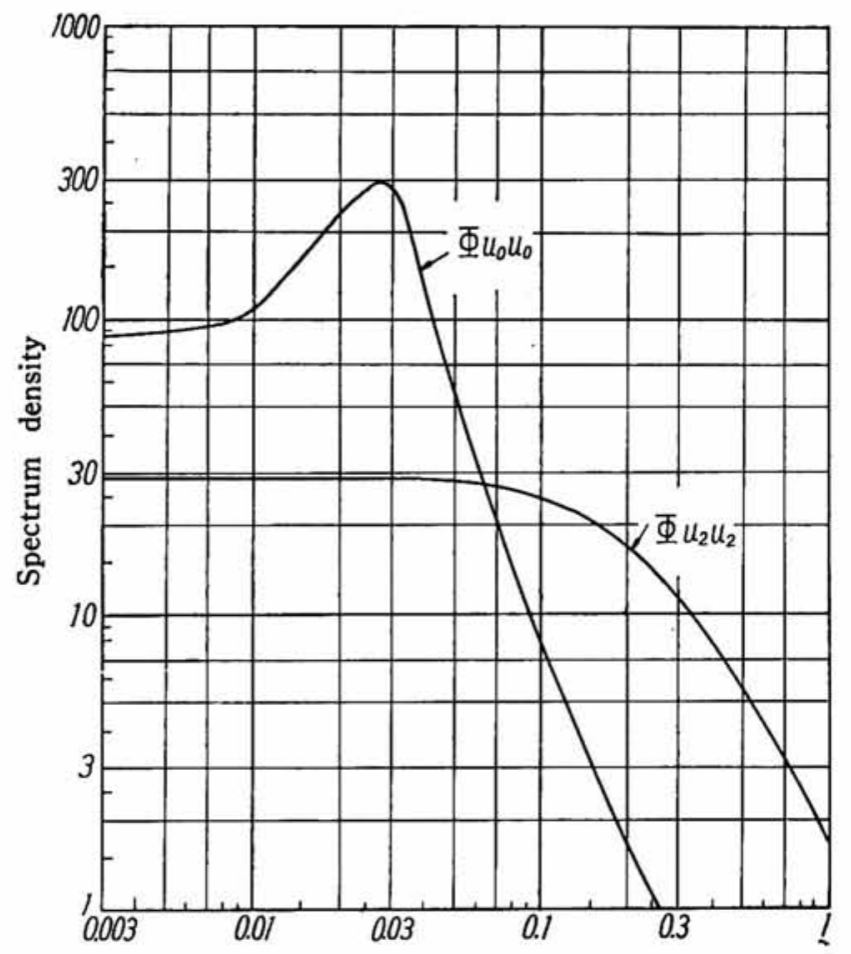

Fig. 21 Spectrum density $\emptyset u_{0} u_{0}$ and $\emptyset u_{2} u_{2}$ as given by eqs. (35) and (36) expotential functions.

$$
\begin{aligned}
& \varphi_{u 2 u 2} \doteqdot 3.62 e^{-0.287 \tau} \\
& \varphi_{u 0 u 0} \doteqdot 3.20 e^{-0.0107 \tau} \cos 0.0271 \tau
\end{aligned}
$$

By substituting eqs. (33) and (34) into eq. (30), autocorrelations $\varphi u_{2} u_{2}$ and $\varphi u_{0} u_{0}$ are converted into spectrum density,

$$
0 u_{2} u_{2}=\frac{29.0}{1+\left(\frac{\omega}{0.25}\right)^{2}}
$$

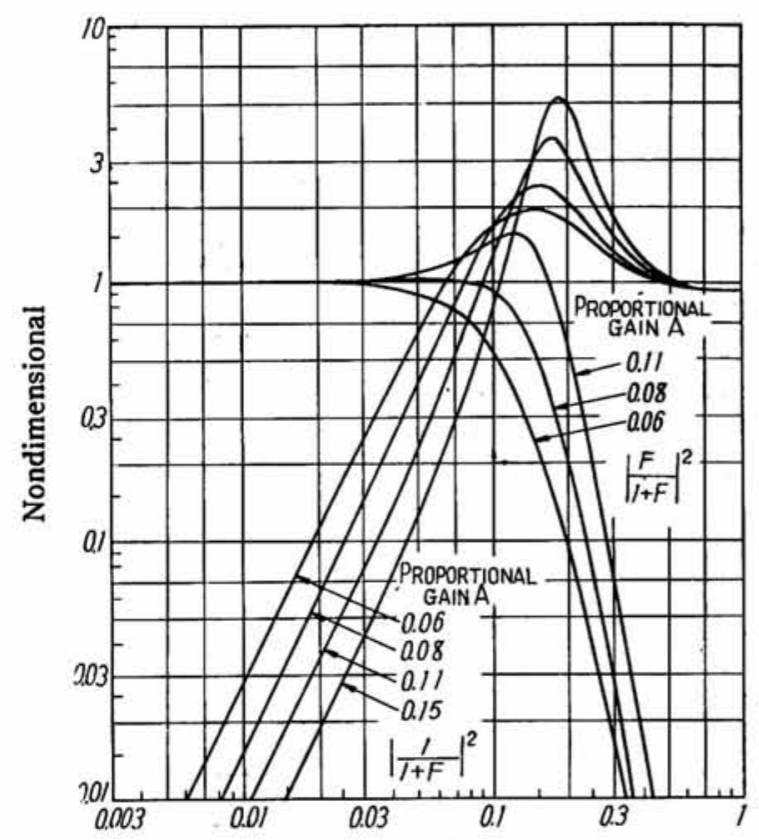

Angular frequency $\omega^{\prime}$ (rad/sec computer time)

Fig. 22 Computed values of factors $\left|\frac{1}{1+F}\right|$ and $\left|\frac{F}{1+F}\right|^{2}$

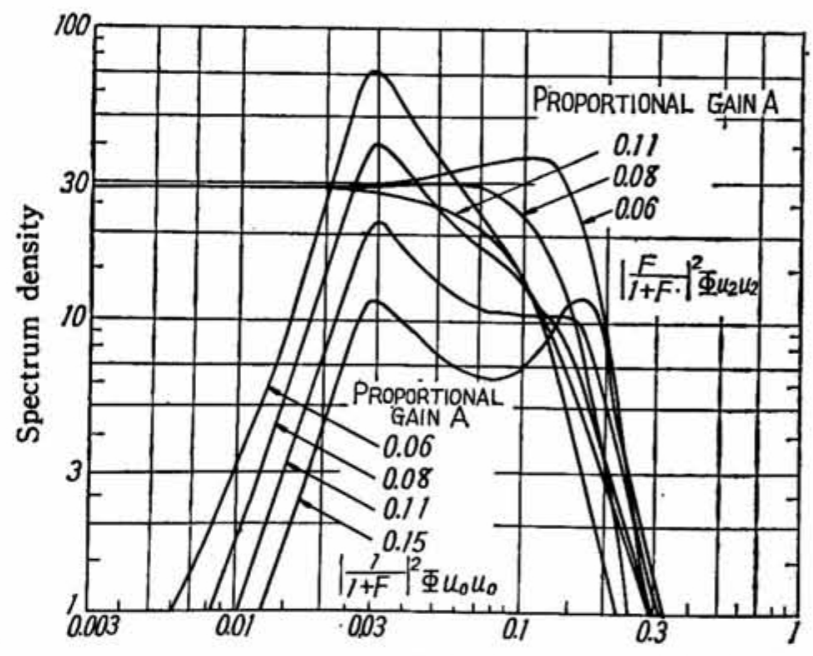

Fig. 23 Computed values of $\left|\frac{1}{1+F}\right|^{2} \oplus u_{0} u_{0}$ and $\left|\frac{F}{1+F}\right|^{2} \bullet u_{2} u_{2}$ 
$\emptyset u_{0} u_{0}=299\left[\frac{1}{1+\left(2.53+93.5 \omega^{\prime}\right)^{2}}+\frac{1}{1+\left(2.53-93.5 \omega^{\prime}\right)^{2}}\right]$

where $\quad \omega^{\prime}=\frac{s^{\prime}}{j}$

$\emptyset u_{3} u_{2}$ and $\emptyset u_{0} u_{0}$ are plotted against $\omega^{\prime}$ on a log-log paper as shown in Fig. 21.

The two factors $|1 / 1+F|^{2}$ and $|F / 1+F|^{2}$ which appear in eq.(32) are readily obtainable graphically by using the vector diagram of $F$ in Fig. 11 . These two factors are computed for four different values of proportional gain $A$ ranging from 0.06 to 0.15 (Figs. 22 and 23).

The sum of the last two terms of eq.(32) can now be readily computed by reading values of $\oplus u_{0} u_{0}$ $\emptyset u_{2} u_{2},|1 / 1+F|^{2}$ and $|F / 1+F|^{2}$ off the diagrams in Figs. 21,22 and 23 . The first term $\emptyset u_{1} u_{1}$ in eq.(32) is irrelevant to the card control since it does not include $F$. In subsequent discussion, therefore, we omit $\nabla u_{1} u_{1}$ from eq.(32) and refer to the sum of the last two terms of eq.(32) in place of $\emptyset_{w w w}$.

The effect of proportional gain $A$ on the mean square value of roving weight variation can be assessed by measuring in Fig. 24 the area sorrounded by each curve and the two axes. The smaller the area, the smaller the estimated mean square variation of roving weight. It is found that $A=0.06$ gives the lowest estimated value of mean square variation.

Just to demonstrate the large influence of the noise $u_{2}$ on the result of card control, $u_{3}$ is arbitrarily assumed to be zero and just the last term of eq.(32) is plotted in Fig. 25.

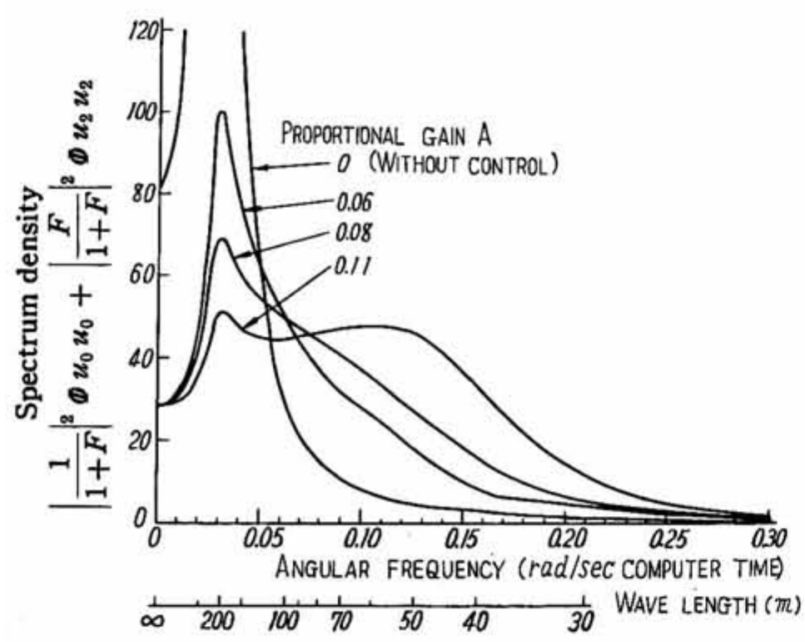

Fig. 24 Computed values of $\left|\frac{1}{1+F}\right|^{2} \oplus u_{0} u_{0}+\left|\frac{F}{1+F}\right|^{2} \circlearrowleft u_{2} u_{2}$
A comparison of Fig. 25 with Fig. 24 reveals a large difference in the magnitude of roving weight variation for the same value of $A$ and an equally large difference in the value of $A$ that makes the mean square variation smallest. $A=0.13$ in Fig. 25 gives the smallest yarn weight variation in contrast to $A=0.06$ in Fig. 24 Fig. 24 and 25 clearly show that the random noise $u_{2}$ generated by the detecting element cannot be overlooked in analysis without causing a grave inaccuracy.

The spectrum density curves in Fig. 24 are computed on the assumption that the card control system has the dynamic characteristic described by the block diagram in Fig. 10 and that the various input signals to the control system have statistical properties as shown by the autocorrelation in Fig. 20 .

To establish the validity of the theoretical reasoning used so far in the present analysis and to check the accuracy of experimental measurements, the spectrum density in Fig. 24 is re-converted into autocorrelations and compared with the autocorrelation derived from the actually measured values of roving weight variation.

The curves in Fig. 24 are, of course, a computed prediction of roving weight variation for the condition that the card is automatically controlled. What we compare with these semitheoretical values are the actual roving weight variation obtained by running the card under automatic control with $R_{t}$ set to $3.4 \mathrm{M} \Omega$ and $R_{f}$ set to $1.2 \mathrm{M} \Omega$.

Substituting eq.(32) into eq.(31), we get,
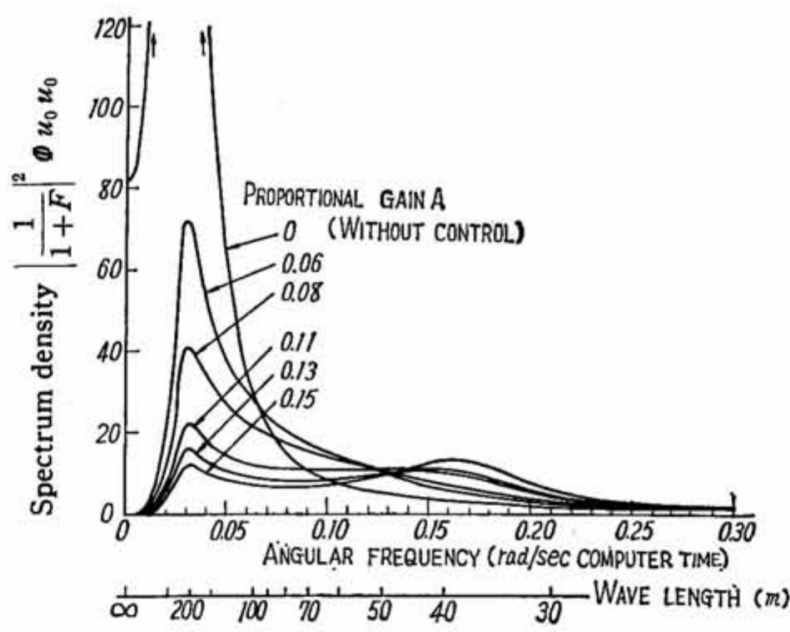

Fig. 25 Computed values of $\left|\frac{1}{1+F}\right|^{2} \oplus u_{0} u_{0}$

Dournal of The Textile Machinery Society of Dapane 


$$
\begin{gathered}
\varphi_{w w}=\varphi u_{1} u_{1}+\frac{1}{2 \pi j} \int_{-j_{\infty}}^{j_{\infty}}\left(\left|\frac{1}{1+F}\right|^{2} \oplus_{0} u_{0} u\right. \\
\left.+\left|\frac{F}{1+F}\right|^{2} \oplus u_{2} u_{2}\right) d s
\end{gathered}
$$

Enclosed in the bracket above is the sum of the last two terms of eq.(32) whose values are plotted in Fig. 24.

There is a graphical method to make computation of eq.(37) on the values given in Fig. 24. Of the several curves in Fig. 24 the one we would like to compare with experimental results is the one for $A=\frac{0.337}{0.34}=0.10$ which corresponds to the abovementioned conditions $R_{f}=1.2 \mathrm{M} \Omega$ and $R_{t}=3.4 \mathrm{M} \Omega$ used in an experimental run of the card under automatic control. The semitheoretical autocorrelation thus derived from the spectrum density in Fig. 24 is curve (b) in Fig. 26. Curve (d) in Fig. 24 is the autocorrelation derived from the experimentally measured values of yarn weight; Curves (b) and (d) share identical values of $R_{t}$ and $R_{f}\left(R_{f}=1.2\right.$ and $R_{l}=3.4$ ).

Although there is some discrepancy between curves (b) and (d), they are close enough to support the experimental and theoretical procedures used in the analysis under review.

The dotted curve (a) in Fig. 26 is similar to (b), except that the value of proportional gain $A$ is the one that gives the smallest mean square value of yarn weight variation $(A=0.06)$. Note here

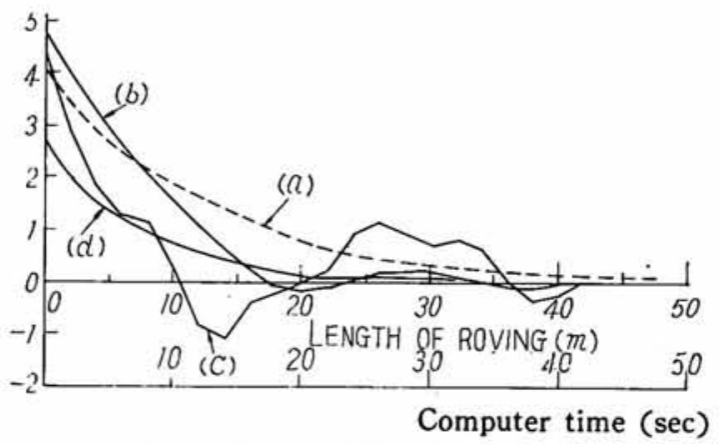

(a) $\varphi_{w w}$ obtained by eq.(37), $A=0.06$

(b) $\varphi_{w r o}$ obtained by eq.(37), $A=0.10$

(c) $\varphi_{\text {wow }}$ computed directly from the measured roving weight.

All conditions correspond to those of (b) above.

(d) Some as (b) above except that $\emptyset u_{2} u_{2}$ assumed to be zero.

Fig. 26 Autocorrelation $\varphi_{v w}$ of roving weight variation, theoretical estimate and experimental that the autocorrelation at its left end is always equal to the mean square value of the time function from which the autocorrelation is derived. On curve (a) which corresponds to the optimum condition $A=0.06$ the mean square value is approximately $4(\%)^{2}$. This means that for the type of input signals shown in Figs. 18 and 20 the woolen card control equipment cannot be expected to reduce the mean square value of yarn weight variation to a value below $4(\%)^{2}$.

The card control equipment actually reduced the mean square variation from $5.2(\%)^{2}$, when uncontrolled (see $\varphi_{z z}$ in Fig. 20), to $4.2(\%)^{2}$ (see curve (d) in Fig. 26) verifying the point made above. The apparent small difference between the two values is presumably due to the fact that the wool fleece fed to the card was so uniform that the effect of automatic control was obscured by the noise $u_{2}$ generated by the measuring element. The decrease in roving weight variation would have been much larger had the wool fleece been highly uneven.

Fig. 24 shows also that the control equipment attenuates long-term variations in roving weight with wave length more than 100 meters, but is not effective on short-term variations.

Curve (d) in Fig. 26 is for a hypothetical case where the measuring noise $u_{2}$ is assumed to be zero. Curve (d) shows a mean square variation of $2.6(\%)^{2}$ which is a highly erroneous prediction of the reality. This fact suggests that in any anaiysis of yarn weight control equipments the effect of noise should always be taken into consideration.

\section{Summary}

The authors conducted a series of field tests on an automatic control equipment, which reduces the roving weight variation, by changing the doffer speed in response to the photoelectrically measured values of web thickness.

The tests consisted of a number of step response tests on the component elements of the control equipment and a separate oscillation test. As a result of these tests, the dynamic characteristics of the control system was described by a mathematical model consisting of a set of transfer functions. Finally statistical metods were used on the abovementioned mathematical model and on various input signals to provide a thoroughly quantitative and analytical picture of the card control system and its effect on the acttually measured values of roving 
weight variation. Some of the results obtained are :

(1) The autocorrelation derived from the actually measured values of roving weight agreed fairly well with the autocorrelation derived from the mathematical model, thereby proving the validity of the theoretical reasoning used in the analysis. (Compare curves (b) and (d) in Fig. 26).

(2) The dynamic characteristics of the card between the doffer speed and the thickness of the web was described by the transfer function

$$
G_{\nu}(s)=\frac{-e^{-12 s}}{1+12 s} \frac{\% \text { change in web thickness }}{\% \text { change in doffer speed }}
$$

indicating the existence of a fairly large time lag in the card.

Loop transfer function of the card control system was found to be

$$
F(s)=\frac{0.075\left(1+8 R_{\mathcal{f}} s\right) e^{-12 s}}{R_{\imath} s(1+10 s)(1+8 s)(1+12 s)}
$$

where potentiometer settings $R_{t}$ and $R_{f}$ are variable over the range $R_{t}=0 \sim 12$ and $R_{f}=0.2 \sim 2.0$.

(3) Random noise generated in the measuring element was found to be of such a magnitude that it materially affected the control result.
(4) Due to the time lag in the card and to the random noise present in the control system, the effectiveness of the control equipment was limited to long-term roving weight variations with wave length over 100 meters. The control equipment was found to be capable of reducing the mean square value of the weight of consecutively cut 2 meter roving samples consisting of 64 roving ends to about $4(\%)^{2}$, or $\sigma=2 \%$.

(5) Test rest results suggest that the statistical methods would also be useful in quantitively analyzing other types of roving weight control systems.

\section{Literature cited}

[1] G.H. Thorndike, R.T.D. Richards, P.D. Jowett ; J. Text. Inst., 48, P 502 (1957-8)

[2] J.G. Truxal: Automatic Feedback Control System Synthesis, 1954, McGraw-Hill

[3] F. Murakami, T. Shoman, S. Kase; Texts for the Twelveth Research Conference, Text. Mach. Soc. Japan (1954)

[4] F. Murakami, T. Shoman, S. Kase; Texts for the Chugoku Area Conference, Text. Mach. Soc. Japan (1961-11) 